This is the final peer-reviewed accepted manuscript of:

Hasan Borke Birgin, Antonella D'Alessandro, Maurizio Favaro, Cesare Sangiorgi, Simon Laflamme, Filippo Ubertini, "Investigation of a low-cost weigh-in-motion vehicle characterization system," Proc. SPIE 11785, Multimodal Sensing and Artificial Intelligence: Technologies and Applications II, 1178505 (20 June 2021)

The final published version is available online at:

https://doi.org/10.1117/12.2593722

Rights / License:

The terms and conditions for the reuse of this version of the manuscript are specified in the publishing policy. For all terms of use and more information see the publisher's website. 


\title{
Investigation of a low-cost weigh-in-motion vehicle characterization system
}

\author{
Hasan Borke Birgin ${ }^{\mathrm{a}}$, Antonella D'Alessandro ${ }^{\mathrm{a}}$, Maurizio Favaro $^{\mathrm{b}}$, Cesare Sangiorgi ${ }^{\mathrm{c}}$, Simon \\ Laflamme $^{\mathrm{d}}$, and Filippo Ubertini ${ }^{\mathrm{a}}$ \\ ${ }^{a}$ University of Perugia Department of Civil and Environment Engineering, via Goffredo Duranti \\ 93, Perugia, Italy \\ ${ }^{b}$ Padana Resine S.r.l., via Calto 731, Ceneselli (RO), Italy \\ ${ }^{\mathrm{c}}$ University of Bologna Department of Civil, Chemical, Environmental, and Materials \\ Engineering, via Zamboni 33, Bologna, Italy \\ ${ }^{\mathrm{d}}$ Iowa State University Department of Civil, Construction, and Environmental Engineering, 416a \\ Town Engr. 813 Bissell Rd., Ames (IA), USA
}

\begin{abstract}
The integration of load tracking systems for structural health monitoring of road infrastructures is a big leap towards future smart cities allowing efficient policy making for sustainable development and fast decision taking with respect to unforeseen events. To facilitate deployment, the load sensing system should be low-cost, easily applicable to any type of road infrastructure, highly durable, and requiring minimum maintenance. In this context, the authors introduce a novel weigh-in-motion (WIM) system for load sensing and vehicle characterization. The integration of the proposed WIM system with machine learning techniques enables the real-time alert of traffic conditions, for example overloads. The sensor is a smart road pavement that generates voltage signals upon the passage of a vehicle. The voltage signals are generated through the piezoresistive effect of the material produced by the inclusion of carbon micro fibers. These signals are processed to generate information about vehicle weight, speed, and class. Preliminary results from field tests show the promise of this technology at WIM sensing.
\end{abstract}

Keywords: weigh-in-motion, pavement-binder, carbon microfiber, self-sensing, piezoresistive, multifunctional materials, traffic sensors, decision making system

\section{INTRODUCTION}

Load sensing systems for civil structures and infrastructures are very beneficial for structural health monitoring (SHM). For example, sensing the traffic loads on bridges may result in improved fatigue life estimation and damage evaluation via predictive SHM models. ${ }^{1}$ Traffic sensors are expected to contribute much to the autonomous sensing and decision making in smart cities. Weigh-in-motion traffic load monitoring is achieved through determination of speed, axles distance, axle separations, gross weight and individual axle weights of the measured vehicle. ${ }^{2}$ Traffic loads can be measured by using piezoelectric sensors, load cells and bending plates, fiber Bratt gratings and through the structural responses of the Bridge, which is also called Bridge-Weigh-in-Motion (BWIM). ${ }^{3,4}$ All the methods listed above are non-optimal because of the following reasons: (i) the sensors are delicate and expensive, (ii) the sensor installation requires skilled worksmanship, (iii) the sensors and the acquisition system require frequent maintenance. For these reasons, traffic monitoring is not yet widely diffused. ${ }^{5}$

An efficient method of traffic load sensing might be by use of smart multi-functional materials, which possess other functionalities aside from load bearing. Piezo-resistivity is one functionality that can be implemented to one structural material. ${ }^{6}$ Literature reports several studies about cementitious materials with enhanced piezoresistive capabilities. ${ }^{7-10}$ Accordingly, the electrical resistance of the cementitious structural elements varies with the strain induced by applied compression loads. The piezoresistive capability is obtained through the addition of conductive

Further author information: (Send correspondence to H.B.B)

H.B.B.: E-mail: hasanborke.birgin@unipg.it 
carbon nano or micro inclusions inside the matrix.Carbon nano inclusions, the carbon particles tend to form clusters inside matrix due to agglomeration. In this case, dispersing agents and sonication of water suspension with fillers are required. As a result, the material is not appropriate for large scaled sensors as demanded by traffic applications. On the other hand, it is possible to effectively disperse carbon micro inclusions inside cementitious matrix by only mechanical mixing, hence sensors with large dimensions can be produced with such materials. ${ }^{11}$

While self-sensing composites with cementitious material matrix are investigated more commonly in the literature, polymer or asphalt material matrices are also studied for strain sensing purposes. ${ }^{12-15}$ Cement paste possess dielectric property that results in polarization under a stable DC voltage. Which causes significant drift in the outcome signal that needs to be removed for a accurate sensing. A method for reducing polarization is adopting biphasic voltage during sensing. ${ }^{16}$ Biphasic current, however, due to the the low acquisition frequency which optimize the outputs, results unsuitable for sensing rapid loads like traffic loads. Moreover, the cementitious sensors are susceptible to the umidity which could affect the electrical results.

As an alternative to the cementitious sensors, this study adopts eco-friendly commercial aggregate binder material called EVIzero for strain sensing pavements. ${ }^{17}$ Carbon microfibers are the fillers adopted for providing electrical conductivity to the material. In this way, pavement material is also facilitated with piezo-resistive properties. The doped EVIzero is a hydrophobic material and has a short initial polarization time when charged under direct current. It allows high resolution measurements with reduced noise levels under various environmental effects, and is highly cost efficient when compared to market available traffic load sensors.

Rest of the paper is organized as follows: section 2 introduces the pavement material and section 3 explains the data acquisition system of the smart pavement. Section 4 presents the results and section 5 discusses the contribution and effectiveness of such a system. Section 6 concludes the paper.

\section{MATERIAL AND SENSOR PRODUCTION}

The novel strain sensing pavement was produced and placed on the factory service road at Padana Resine s.r.l., Rovigo, Italy. The materials adopted in this study are eco-friendly aggregate binder EVIzero by Corecom s.r.l., Ancona Bianco aggregates (agg.) by SINTEX s.r.l, Ancona, Italy, SIGRAFIL carbon microfibers (CMF) by SGL Carbon and twelve 3-meter long copper wires with 1-mm diameter. The main characteristics of the used materials are summarized in the Table 1.

Table 1. Key features of smart pavement materials: EVIzero, ${ }^{17} \mathrm{CMF}^{18}$ and copper.
\begin{tabular}{l|c|c|c} 
feature & EVIzero & $\mathrm{CMF}$ & copper \\
\hline conductivity $\left(\mathrm{S} \cdot \mathrm{m}^{-1}\right)$ & - & $6.67 \cdot 10^{4}$ & $58.7 \cdot 10^{6}$ \\
density $\left(\mathrm{gr} / \mathrm{cm}^{3}\right)$ & 0.85 & 1.80 & 8.96 \\
melting point $\left({ }^{\circ} \mathrm{C}\right)$ & 75 & 3600 & 1085 \\
mixing temperature $\left({ }^{\circ} \mathrm{C}\right)$ & $160-170$ & - & - \\
dynamic viscosity at $160^{\circ} \mathrm{C}(\mathrm{mPa} \cdot \mathrm{s})$ & 700 & - & - \\
\hline
\end{tabular}

The manufactured smart pavement is $150 \times 300 \mathrm{~cm}^{2}$, longitudinal and transversal to the traffic direction, respectively; with a thickness of $8 \mathrm{~cm}$. The density of the compacted pavement sample is $2.21 \mathrm{~g} / \mathrm{cm}^{3}$, prepared using the mix design reported in Table 2.

Table 2. Mix design of the smart pavement volume with the dimensions of 300x150x8 $\mathrm{cm}^{3}$.

\begin{tabular}{|c|c|c|c|c|c|}
\hline components & \multicolumn{3}{|c|}{ aggregates (Ancona Bianco) } & EVIzero & $\mathrm{CMF}$ \\
\hline size & $0-4 \mathrm{~mm}$ & $4-8 \mathrm{~mm}$ & $8-12 \mathrm{~mm}$ & & \\
\hline weight $(\mathrm{kg})$ & 225 & 413 & 112 & 45 & 0.5 \\
\hline weight ratio to total agg. weight(\%) & 30 & 55 & 15 & 6 & 0.066 \\
\hline
\end{tabular}

The layout of the sensing pavement is presented in Figure 1. The pavement sensor includes 12 electrodes divided in 4 groups of electrodes are formed with 3 electrodes, where central electrodes placed at a distance of 30 $\mathrm{cm}$, as shown in Figure 1. 


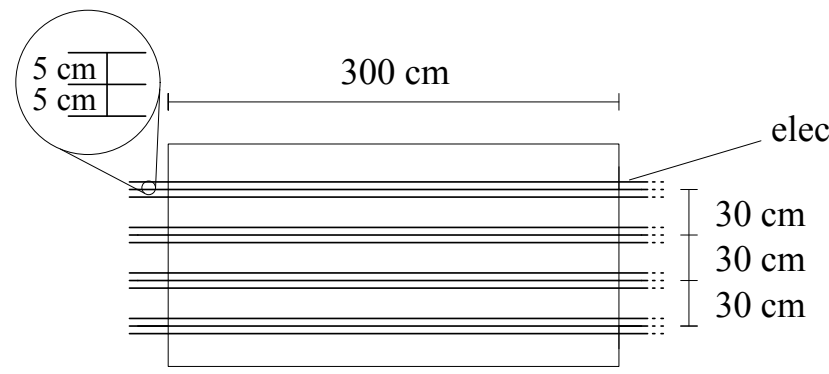

(a) electrodes

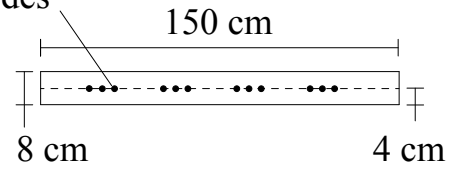

(b)

Figure 1. The electrodes layout of the smart pavement section made of EVIzero; (a) top-view of the pavement; (b) side-view of the pavement.

The electrodes were placed in the middle of the thickness by use of an electrode holding frame (Figure 2(a)). It was build by 4 wood profiles with a section of $2 \times 1 \mathrm{~cm}^{2}$, forming a rectangle of 120x300 $\mathrm{cm}$. Wood profiles were suitable to the purpose since wood does not alter the electrical properties of the pavement and is resistant to the compaction temperature. The electrodes were soldered to electrical cables connected to DAQ and covered by heat resistant tapes. After placement to desired location, the lateral wood profiles were removed from the frame, to ensure an uniform material distribution inside the pavement section. The electrode frame and the final state of the field implementation, are shown in Figure 2(a,b).

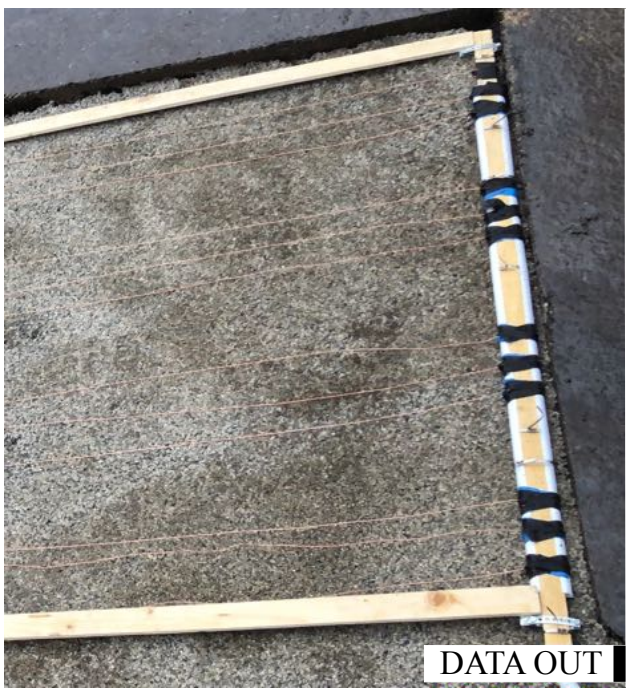

(a)

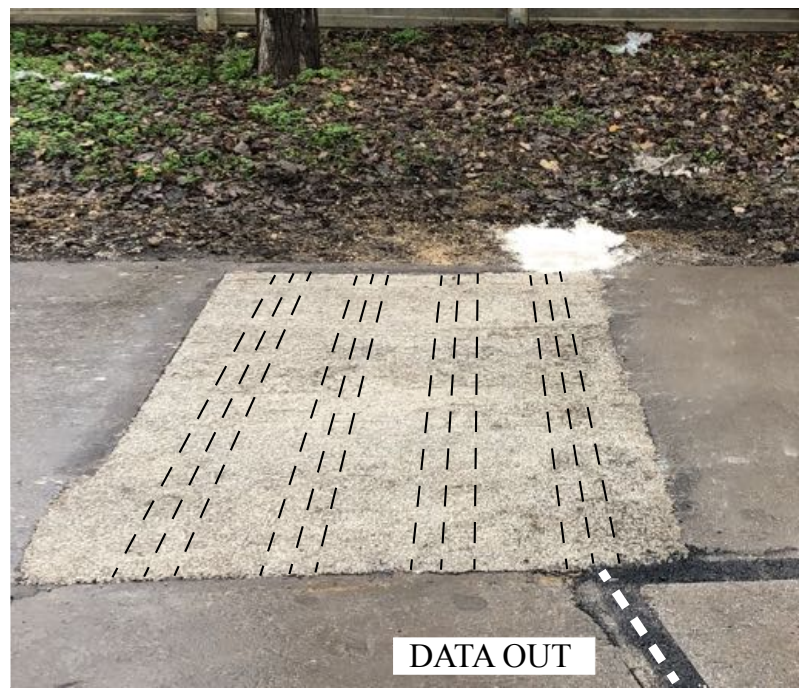

(b)

Figure 2. The pictures of field implemenation (a) electrode holding frame in detail, the DAQ connection is labeled by "data out"; (b) final state of the field setup, the directions of the electrodes are indicated by black dashed lines, the DAQ connection is shown by white dashed line.

\section{INSTRUMENTATION AND VEHICLE LOADS}

The smart pavement sensor is able to monitor the traffic loads thanks to the piezo-resistive effect. When an axle of vehicle passes over the sensing pavement, the electrical resistance of the pavement varies proportionally to the weight of the axle. Voltage input-output units by National Instruments have been used for sensing such a variation. The circuit was formed by a shunt resistor and different sections of the smart pavement connected in series. The circuit has been powered by voltage output source NI-PXIe-4138 with 3 volts of DC. This voltage value was selected to be in accordance with the commonly used levels in recent electronics. During the measurements, the voltage differences through the shunt resistor and smart pavement were measured simultaneously. The resistance 
time history of the smart pavement was then calculated by employing Ohm's law. For the monitoring of the vehicles, two different configurations of smart pavement have been used as presented in Figure 3.

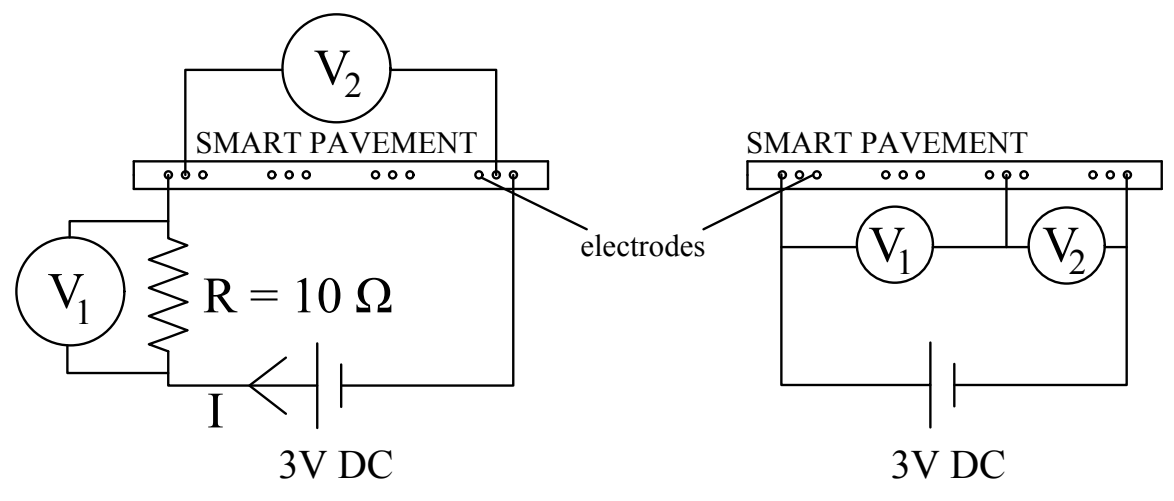

(a)

(b)

Figure 3. The adopted circuit models for different types of sensing; (a) utilization of whole body as sensor for general load sensing; (b) differential reading for wave-form generation.

Accordingly, the circuit presented in Figure 3(a) uses whole pavement for axle weight measurements. The another approach is presented in Figure 3(b), where two halves of the pavement were connected as resistors in series and respective voltage differences were measured. This type of sensing has been developed to produce scalable wave-form during vehicle passing, in accordance to the reference study. ${ }^{6}$

The tests were carried out during the passages of the trucks on the factory service road. Trucks weights were variable due to their loading state: the unloaded trucks weighed between 15-20 tons and loaded trucks weighed between 38-45 tons. Trucks had 5 axles where the last three are closely placed. The weight information of the trucks was taken from the company, because the trucks were weighted by a static scale of the factory near the test field. Further tests with different load scenarios were conducted with three vehicles of different weights: (i) Car1 with a gross weight of $1800 \mathrm{~kg}$, (ii) Car2 with a gross weight of $1200 \mathrm{~kg}$, (iii) Forklift with a gross weight of $4660 \mathrm{~kg}$.

\section{ELECTRICAL PROPERTIES AND MEASUREMENT RESULTS}

\subsection{Electrical characteristics}

Prior to the vehicle monitoring tests, initial electrical measurements were conducted to assess the electrical properties of the pavement. Equation 1 defines the linear relation between resistivity, $\rho$, electrical resistance, $R$ :

$$
R=\rho \frac{L}{A}
$$

where crossectional area $A$ is $2400 \mathrm{~cm}^{2}$ and $L$, distance between electrodes, is measured with respect to the initial electrode. The linear growth was examined through the resistance readings between different electrodes and readings are plotted in Figure 4. 


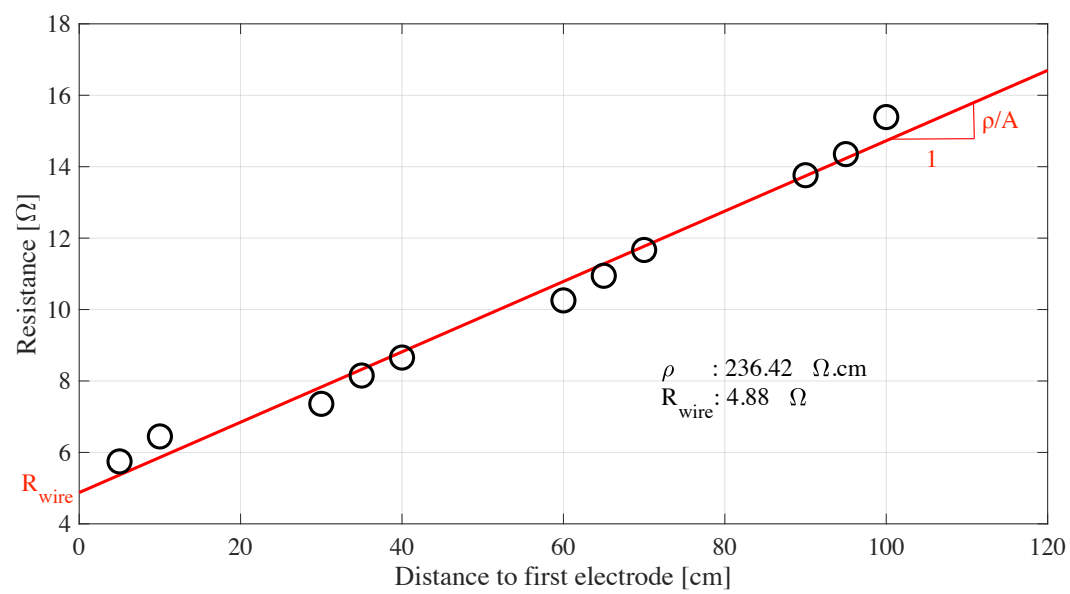

Figure 4. The resistance growth inside the material and the linear model with respect to the distance from initial electrode. The resistance readings between initial and the electrodes at specified locations are indicated with circles, the linear fit line of resistance model is plotted in red.

Obtained results demonstrated that smart pavement's conductivity was not affected by the surrounding asphalt. From the tests, the resistivity of the pavement sample was found as, $\rho=236.4 \Omega \cdot \mathrm{cm}$. The resistance induced by the DAQ cables was found approximately of $5 \Omega$. Being consistent with the linear resistance model, initial electrical readings verified the one directional current flow inside the pavement and uniform dispersion of carbon fibers, demonstrated the reliability and consistency of vehicle measurements.

\subsection{Tests with vehicles}

\subsubsection{Forward pass of three vehicles}

First campaign of tests were carried out by passing over the sensing pavement with the three types of vehicles presented in previous sections. Figure 5 presents the collected data from the pavement, during the passages.

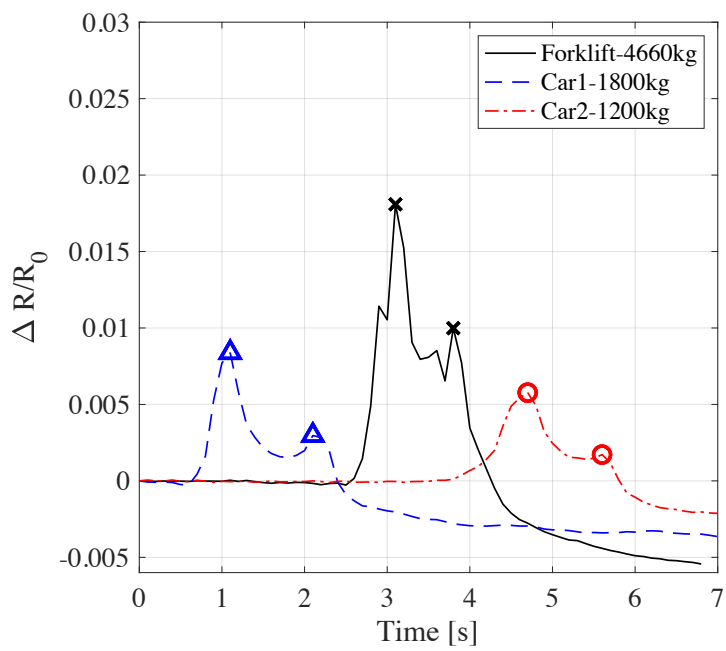

(a)

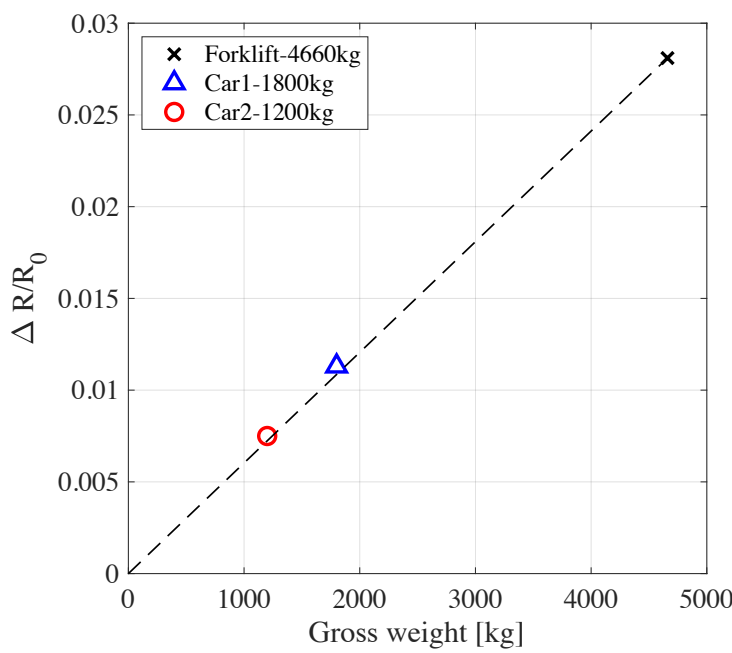

(b)

Figure 5. Forwards passings of three different vehicles and their signal responses in terms of fractional variation of resistance time history: (a) the generated signals; (b) the linear model produced by summation of peak values reached by the both axles of vehicles.

Accordingly, the observed amplitudes of the generated signal by the pavement were approximately proportional to the weight of the vehicles passing. The relation was close to linear as demonstrated in Figure 5(b), where the 
summation of peak values of axle occurrences in Figure 5(a) are plotted together with gross weights. Moreover, the weight differences between front and rear axles are also observable, that is caused by the presence of motor in front of the cars.

\subsubsection{Forward and backward passes of two different cars}

Sequential forwards and backwards passing was performed with the two cars of different weights. The aim was to prove that the response of the pavement to the axle weights didn't depend on the load direction, and was repeatable. The two time histories with the similar load pattern are shown in Figure 6.

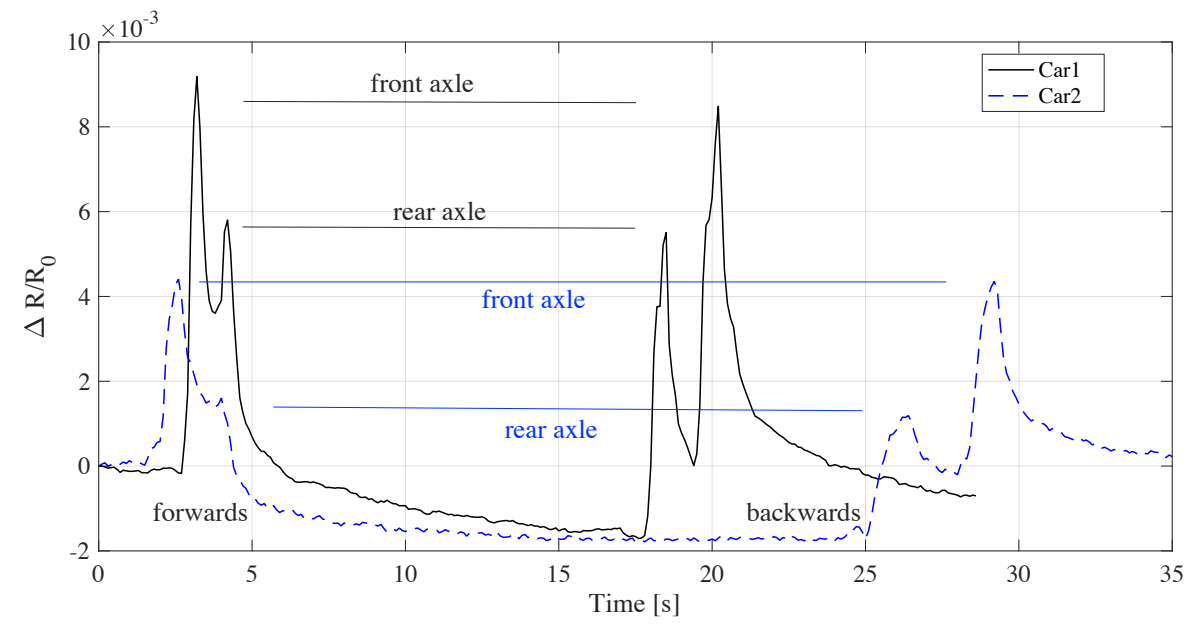

Figure 6. Consecutive forwards and backwards passings of two cars and their signal responses in terms of fractional variation of resistance time history.

The both time histories shows similar responses to the load pattern with magnitudes scaled to the vehicle weights. Moreover, the similar values were obtained from the passages forward and backwards of the same axles.

\subsubsection{Wave-form shaped time histories for WIM characterization algorithm}

As proposed in reference study, ${ }^{6}$ signals that are useful for WIM characterization can be obtained via adopting the sensing case in Figure 3(b). Car2 passed over the pavement in different ways in order to explore the suitability of the obtained resistance time histories: (i) forwards pass, (ii) backwards pass, (iii) front axle passes subsequently backwards and forwards. The obtained signal time histories are presented in Figure 7.

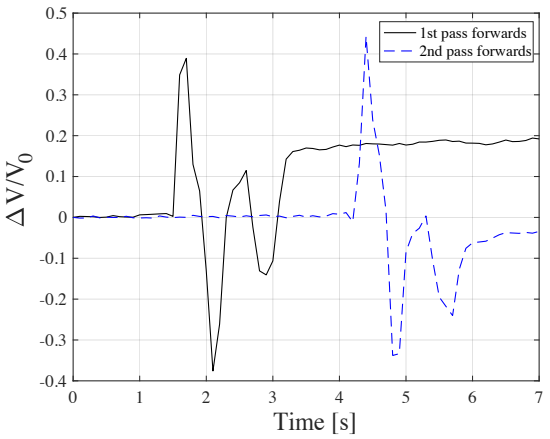

(a)

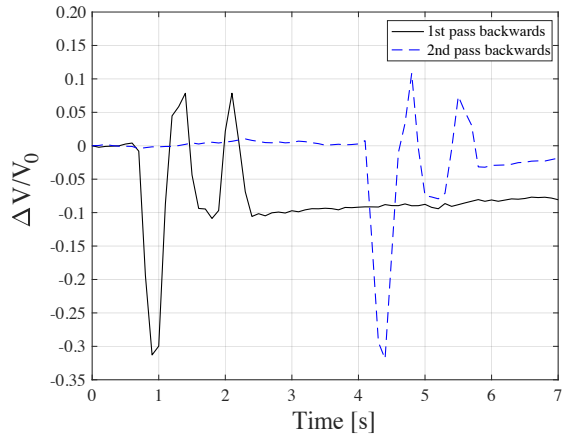

(b)

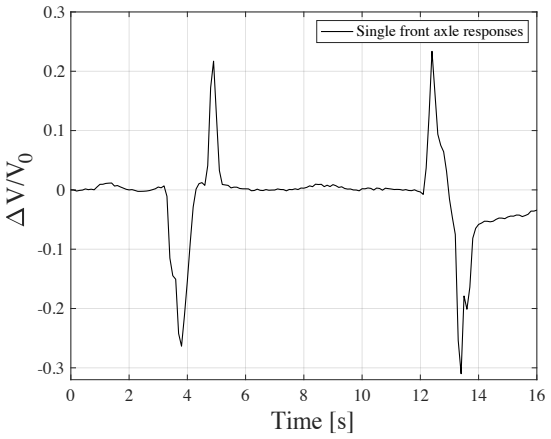

(c)

Figure 7. The outcomes of wave-form generation with Alfa-Romeo Mito; (a) forwards pass repeated twice; (b) backwards pass repeated twice; (c) successive pass of front axle backwards and forwards, respectively. 


\subsection{Records of 5-axle trucks}

During the recordings trucks passed with a constant speed. The weights of the trucks were later measured by the truck scale, located near the field test. The signals coming from different trucks are presented in the Figure $8(\mathrm{a})$.

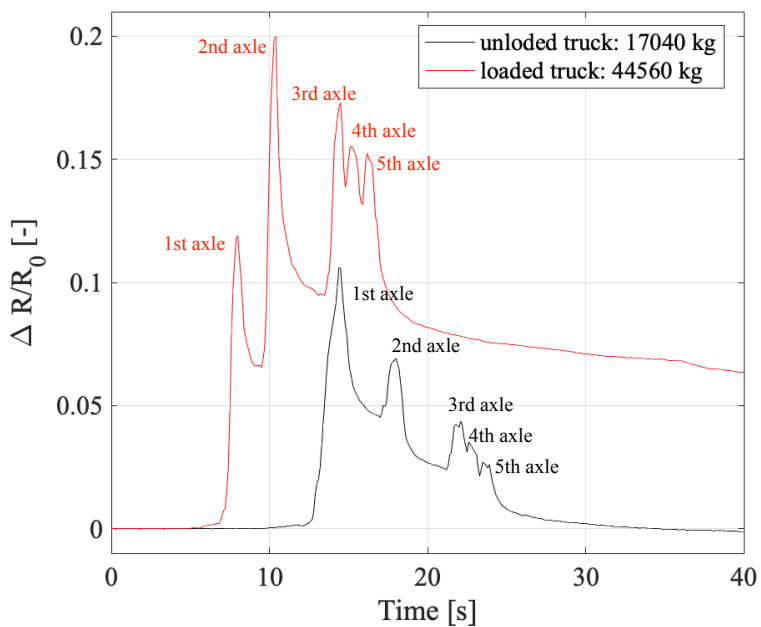

(a)

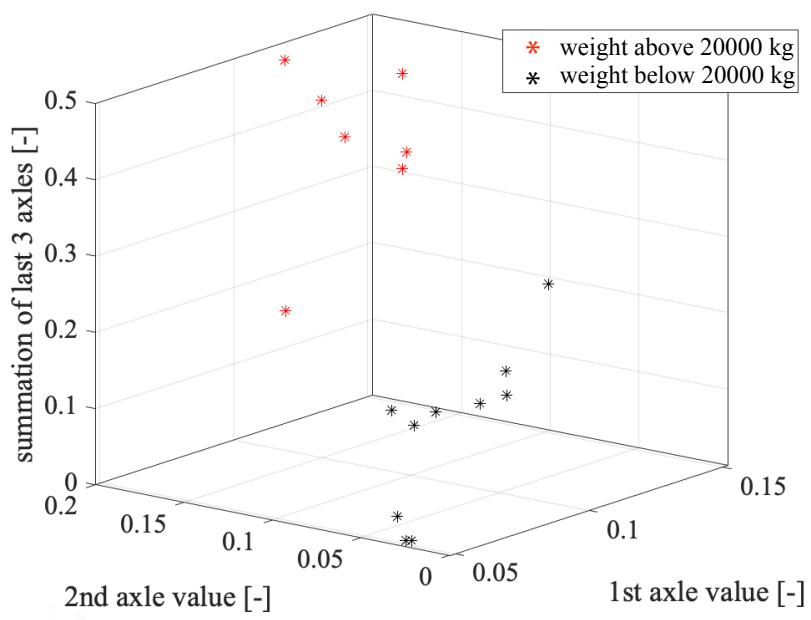

(b)

Figure 8. The collected data from truck passings. (a) Typical signals obtained from a loaded and unloaded truck; (b) peak values from axle passings and the classification according to the threshold of $20000 \mathrm{~kg}$. The values coming from last 3 axles are presented in their summation in the z-coordinate.

Accordingly, the signals were found reflecting the characteristics of the trucks. The presence and weight distributions of the axles were clearly visible and distinguishable within the acquired signals. The obtained peak values are plotted in 8(b), together with a classification of corresponding gross weights of truck with a threshold of $20000 \mathrm{~kg}$. It was observed that, two classes were distinctive and thus suitable for autonomous decision systems.

\section{DISCUSSION}

The results of the experimental campaign shows that, piezo-resistive smart materials are quite functional for monitoring the traffic load and WIM. Based on the results, following statements could be discussed: (i) the electrical characteristics of the pavement are uniform, based on the resistance readings between various electrodes in different sections. The dispersion of the carbon fibers is homogeneous inside the pavement. (ii) Moreover, the DAQ system is effective with only $3 \mathrm{~V}$ of applied voltage, which results efficient in terms of power consumption. (iii) The designed load sensing pavement is sensitive to wide range of weights. The gross weight range of the vehicles in this study is from $1200 \mathrm{~kg}$ to $42000 \mathrm{~kg}$. (iv) The pavement response is scalable to the vehicle weights and repeatable. The obtained signal magnitudes don't depend on the travel direction of the vehicle. (v) The pavement is capable to generate the WIM characterization signals. The noise and chattering within these type of signals are observed to be low with respect to wave amplitudes generated by the vehicles, this characteristics make the novel type of sensing pavement promising for creation of low cost WIM system.

\section{CONCLUSION}

In this study, the field application of a strain sensing pavement made of piezo-resistive smart material and the obtained preliminary about its traffic monitoring capabilities are investigated. According to the results, weight sensitive, repeatable and clean signals in terms of noise are attained. The obtained signal characteristics reflect the vehicle geometries, and, the smart pavement is capable of producing the theoretical WIM characterization wave-forms of reference study. The electrical properties of the pavement are stable and current flow can be 
assumed as one directional. The initial results of the field tests point out that the smart pavement appear suitable for detecting traffic loads and providing weigh-in-motion monitoring. Their application and maintenance result easier and with lower costs with respect to traditional road monitoring systems.

\section{ACKNOWLEDGMENTS}

This research has received funding from the European Union's Horizon 2020 research and innovation programme under Grant Agreement N. 765057 - SAFERUP! Project.

\section{REFERENCES}

[1] Maljaars, J., "Evaluation of traffic load models for fatigue verification of european road bridges," Engineering Structures 225, 111326 (2020).

[2] Jacob, B. and Feypell-de La Beaumelle, V., "Improving truck safety: Potential of weigh-in-motion technology," IATSS research 34(1), 9-15 (2010).

[3] Lydon, M., Taylor, S. E., Robinson, D., Mufti, A., and Brien, E., "Recent developments in bridge weigh in motion (b-wim)," Journal of Civil Structural Health Monitoring 6(1), 69-81 (2016).

[4] Yu, Y., Cai, C., and Deng, L., "State-of-the-art review on bridge weigh-in-motion technology," Advances in Structural Engineering 19(9), 1514-1530 (2016).

[5] Haugen, T., Levy, J. R., Aakre, E., and Tello, M. E. P., "Weigh-in-motion equipment-experiences and challenges," Transportation Research Procedia 14, 1423-1432 (2016).

[6] Birgin, H. B., Laflamme, S., D'Alessandro, A., Garcia-Macias, E., and Ubertini, F., "A weigh-in-motion characterization algorithm for smart pavements based on conductive cementitious materials," Sensors 20(3), 659 (2020).

[7] Downey, A., D’Alessandro, A., Ubertini, F., and Laflamme, S., "Automated crack detection in conductive smart-concrete structures using a resistor mesh model," Measurement Science and Technology 29(3), 035107 (2018).

[8] D’Alessandro, A., Ubertini, F., Laflamme, S., Rallini, M., Materazzi, A. L., and Kenny, J. M., "Strain sensitivity of carbon nanotube cement-based composites for structural health monitoring," in [Sensors and Smart Structures Technologies for Civil, Mechanical, and Aerospace Systems 2016], 9803, 980319, International Society for Optics and Photonics (2016).

[9] Han, B., Ding, S., and Yu, X., "Intrinsic self-sensing concrete and structures: A review," Measurement 59, 110-128 (2015).

[10] Lee, S.-J., You, I., Zi, G., and Yoo, D.-Y., "Experimental investigation of the piezoresistive properties of cement composites with hybrid carbon fibers and nanotubes," Sensors 17(11), 2516 (2017).

[11] Birgin, H. B., D'Alessandro, A., Laflamme, S., and Ubertini, F., "Smart graphite-cement composite for roadway-integrated weigh-in-motion sensing," Sensors 20(16), 4518 (2020).

[12] Liu, X., Wu, S., Li, N., and Gao, B., "Self-monitoring application of asphalt concrete containing graphite and carbon fibers," Journal of Wuhan University of Technology-Mater. Sci. Ed. 23(2), 268-271 (2008).

[13] Liu, X. and Wu, S., "Study on the graphite and carbon fiber modified asphalt concrete," Construction and Building Materials 25(4), 1807-1811 (2011).

[14] Liu, X., Nie, Z., Wu, S., and Wang, C., "Self-monitoring application of conductive asphalt concrete under indirect tensile deformation," Case Studies in Construction Materials 3, 70-77 (2015).

[15] D'Alessandro, A., Coffetti, D., Crotti, E., Coppola, L., Meoni, A., and Ubertini, F., "Self-sensing properties of green alkali-activated binders with carbon-based nanoinclusions," Sustainability 12(23), 9916 (2020).

[16] Downey, A., D'Alessandro, A., Ubertini, F., Laflamme, S., and Geiger, R., "Biphasic dc measurement approach for enhanced measurement stability and multi-channel sampling of self-sensing multi-functional structural materials doped with carbon-based additives," Smart Materials and Structures 26(6), 065008 (2017).

[17] Corecom, "Evizero technical data sheet." https://www.evizero.com/download/ technical-data-sheet-evizero.pdf. Accessed: 2021-02-25.

[18] Carbon, S., "Sigrafil short carbon fibers." https://www.sglcarbon.com/en/markets-solutions/ material/sigrafil-short-carbon-fibers. Accessed: 2021-02-25. 